\title{
A New Metamorphic Parallel Leg Mechanism with Reconfigurable Moving Platform
}

\author{
Yong Xu $\mathbb{D}$, Zheng Liang, and Jiali Liu \\ School of Mechanical and Automotive Engineering, Shanghai University of Engineering, Science, Shanghai 201620, China \\ Correspondence should be addressed to Yong Xu; brucexuyong@163.com
}

Received 11 December 2019; Accepted 10 February 2020; Published 14 March 2020

Academic Editor: Alessandro Gasparetto

Copyright (c) 2020 Yong Xu et al. This is an open access article distributed under the Creative Commons Attribution License, which permits unrestricted use, distribution, and reproduction in any medium, provided the original work is properly cited.

\begin{abstract}
This paper proposes the concept of full configuration state of metamorphic mechanism. Based on the concept, the configuration synthesis principle of metamorphic parallel mechanism is put forward. Firstly, a metamorphic parallel mechanism in full configuration state is synthesized, and then full configuration state evolves into a specific configuration state by increasing constraints or decreasing degrees of freedom. A reconfigurable moving platform based on the triple symmetric Bricard spatial closed-loop mechanism with a single degree of freedom is proposed. Based on this, a new method for switching motion configuration states of the metamorphic parallel mechanism is constructed. According to the configuration synthesis principle presented above, a novel metamorphic parallel mechanism that can switch between three- and four-degree-of-freedom is synthesized, and then the triple symmetric Bricard spatial closed-loop mechanism is used as the reconfigurable moving platform (that is, the reconfigurable foot of a walking robot) of the metamorphic mechanism, and thus, a novel metamorphic parallel leg mechanism is created. The screw theory is used to verify the degrees of freedom of the new type of metamorphic parallel leg. The proposed metamorphic parallel leg mechanism is expected to improve flexibility and adaptability of walking robots in unstructured environment.
\end{abstract}

\section{Introduction}

The mechanism whose topological configuration and degree of freedom can change is called the metamorphic mechanism due to changes in the number or characteristics of components and kinematic pairs during the continuous motion of the mechanism [1]. The essential differences between metamorphic mechanism and conventional mechanism are that the former has variable topological configurations and degrees of freedom and has the characteristic of evolution among different topological configurations and degrees of freedom [2]. In this paper, the motion state corresponding to the specific topological configuration and degree of freedom of a metamorphic mechanism is called the "configuration state" of the mechanism.

The configuration synthesis of parallel mechanism is embodied in the process of deriving the configurations of all branches from the degree-of-freedom requirement of the mechanism and then assembling branches into a parallel mechanism. The full configuration state (denoted as "s configuration state") of the metamorphic mechanism in this paper is defined as the configuration state which can meet all the requirements of degrees of freedom. The full configuration state of metamorphic mechanism is the initial configuration state of the mechanism, which can further evolve into other specific configuration states. Therefore, the full configuration state is sure to contain information about the topological configurations and degrees of freedom of all other configuration states of the mechanism.

There are generally five ways of transforming topological configurations and degrees of freedom of metamorphic mechanism [3], that is, changing the number of components, changing the number of kinematic pairs, changing the characteristics of components, changing the characteristics of kinematic pairs, and changing the adjacency relationship between component modules. Among them, the number of components is changed by combination and separation of 
components, the number of kinematic pairs changed by locking and releasing of kinematic pairs, the characteristics of components changed by different stiffnesses or flexibilities, the characteristics of kinematic pairs changed by different types and axes geometric relationship, and the adjacency relationship between component modules changed by reorganization of the same or similar units.

Compared with serial mechanism, parallel mechanism has advantages such as good motion stability, large structural rigidity/strength, and high motion precision [4]. The metamorphic parallel mechanism, which combines advantages of metamorphic mechanism and parallel mechanism, overcomes shortcomings of fixed freedoms and few configuration states of conventional parallel mechanism and satisfies demands such as multifunction, high precision, high stability, high adaptability, and low cost in the era of intelligent manufacturing; therefore, the metamorphic parallel mechanism undoubtedly has great academic value and broad industrial prospects.

At present, research hotspots of parallel metamorphic mechanisms mainly focus on configuration synthesis and metamorphic characteristic analysis. Wang et al. [5] proposed the concept of metamorphic source matrix and corresponding construction method, established mathematical model of the evolution law of metamorphic mechanism in different motion modes, and simplified the configuration synthesis of metamorphic mechanism into that of multimotion-mode mechanism. Xi et al. [5] designed a parallel metamorphic mechanism that changes the number of degrees of freedom of the mechanism through connection or separation of swing and sliding modules in branches and moving platform of the mechanism. Ye et al. [6] proposed a configuration synthesis method of parallel metamorphic mechanism through different combinations of basic branch units with lockable kinematic pairs. Gan [7] proposed the $r R$ metamorphic revolute pair with variable axial positions and the rT metamorphic kinematic pair with two configurations of hooke hinge and revolute pair [8]. Based on these two kinds of metamorphic kinematic pairs, a variety of metamorphic parallel mechanisms are designed. Kong et al. [9] presented a three-degree-of-freedom parallel metamorphic mechanism with multiple motion modes based on a reconfigurable moving platform with lockable joints. Liu Jiangnan [10] proposed a new configuration design method of metamorphic mechanism based on constraint variation characteristics of metamorphic kinematic pairs.

Parallel mechanisms with constant degree of freedom and topological configuration have been widely applied in leg mechanisms of walking robots [11], and such leg mechanisms have poor motion flexibility and adaptability in complex unstructured environment. In view of this, this paper proposes a novel metamorphic parallel leg mechanism with a reconfigurable foot. The reconfigurable foot is actually the moving platform of the metamorphic parallel mechanism, and its topology configuration is a reconfigurable spatial closed-loop linkage mechanism. The reconfigurable foot can transform its shape which is driven by the actuator of the spatial closed-loop mechanism, thus enabling flexible switching of foot-to-ground contact states, improvement of walking stability, and various configuration states required by different terrains.

Based on the constraint screw theory [12], in this paper, the logical relations among the constraint screw systems of metamorphic parallel leg mechanism in various configuration states are deduced, and according to these logical relations, the principle of configuration synthesis of metamorphic mechanism is proposed. Based on the independent drive characteristic of the triple symmetric single-degree-offreedom Bricard mechanism (that is, the reconfigurable moving platform), a multiconfiguration-state switching method for the metamorphic parallel mechanism is proposed, which is expected to improve flexibility and adaptability of walking robots in unstructured environment.

\section{Analysis of Degree of Freedom for Metamorphic Parallel Leg Mechanism}

To realize discontinuous contacts with three-dimensional terrains for a walking robot, a walking leg mechanism should have at least three spatial degrees of freedom, which moreover should include at least one prismatic degree of freedom in order to adapt to the terrain well and decrease foot-ground impact [13].

A mammalian leg generally contains two rotational degrees of freedom around the hip joint and one translational degree of freedom [13]. The leg with such 2R1T (where $R$ denotes a rotational degree of freedom and $T$ denotes a translational degree of freedom) degrees of freedom is easy to control and can walk quickly but is not flexible enough to turn; it therefore may lead to the overall instability of the robot. If the leg has one more rotational degree of freedom (the leg now has 3R1T degrees of freedom), the small side swing generated by the robot body during turning can be adjusted [14], thus improving walking flexibility and stability of the robot.

Aiming at the requirements of degrees of freedom for walking robots in complex and variable environments, this paper proposes a new concept of metamorphic parallel leg mechanism with a reconfigurable moving platform (as the reconfigurable foot of the robot). The metamorphic parallel leg mechanism has two kinds of degrees of freedom and corresponding configuration states: when the robot walks on flat terrain, each leg adopts the configuration state of 2R1T, as shown in Figure 1; when it walks on complex terrain (such as steps), the leg adopts the configuration state of 3R1T, as shown in Figure 2.

\section{Principle of Configuration Synthesis of Parallel Metamorphic Mechanism}

To meet different requirements of degrees of freedom, a metamorphic mechanism has multiple configuration states, and each configuration state has specific number and type of degree of freedom. During continuous motion of metamorphic mechanism, a specific configuration state of the mechanism can evolve to another one via the unique full configuration state. 


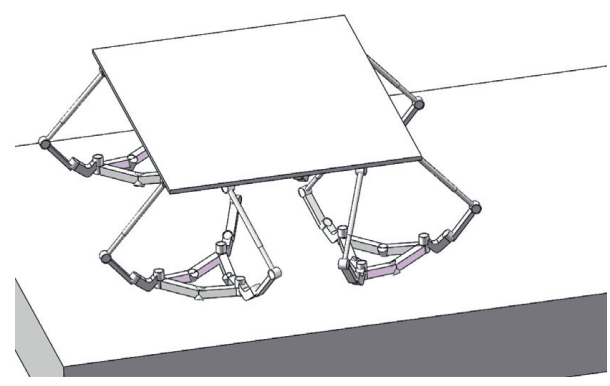

(a)

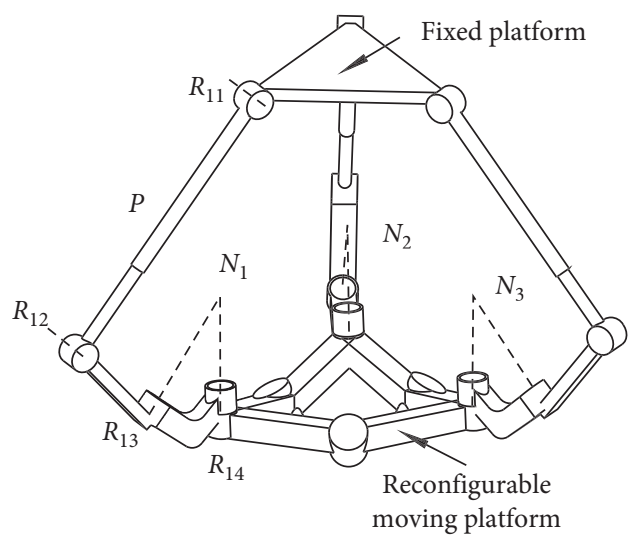

(b)

FIGURE 1: Walking robot with (a) 2R1T legs and (b) 2R1T parallel leg mechanism.
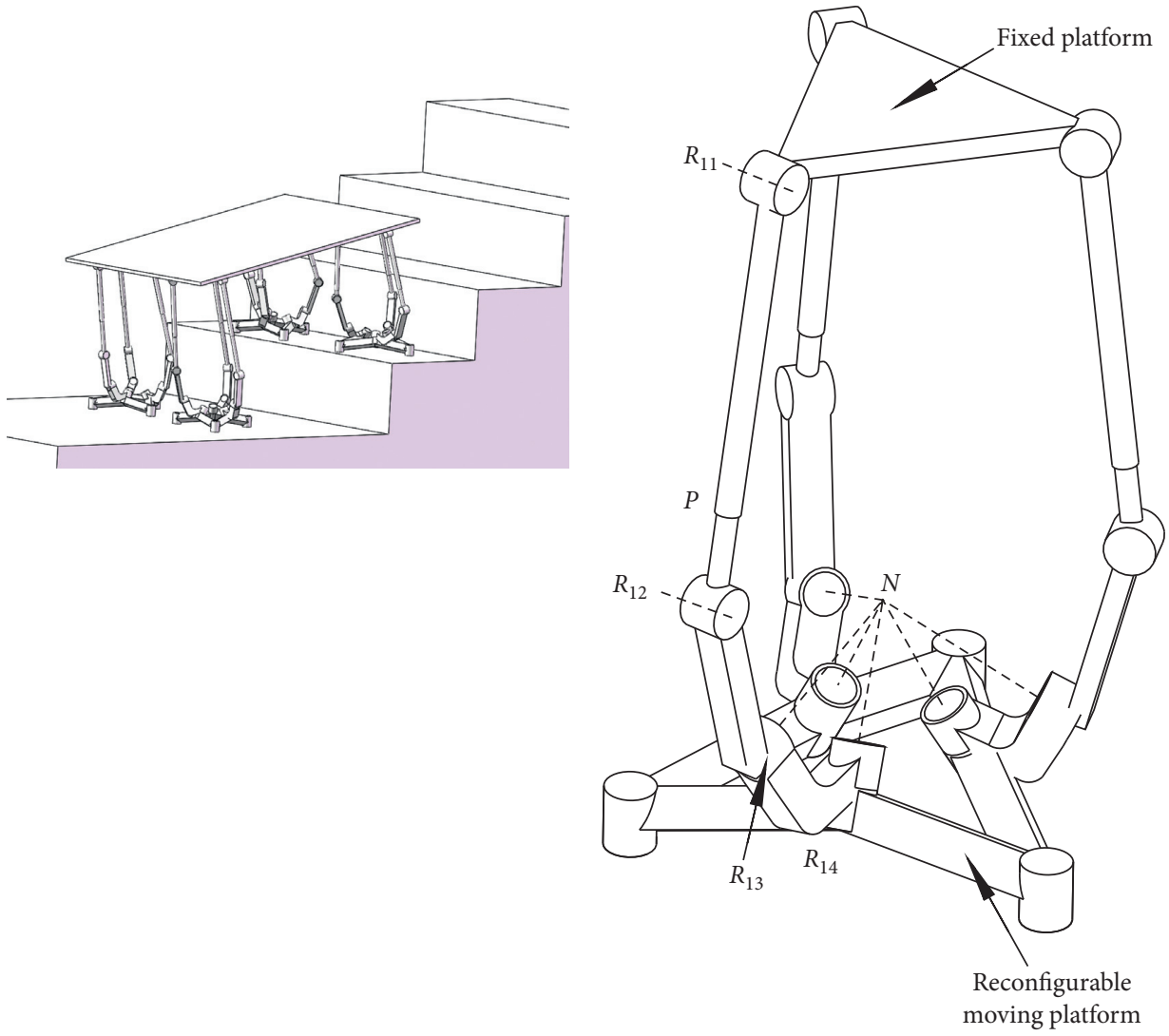

(a)

(b)

FIgURE 2: Walking robot with (a) 3R1T legs and (b) 3R1T parallel leg mechanism.

It can be seen that the key problem of configuration synthesis of metamorphic mechanism is to firstly synthesize the mechanism in full configuration state, which will further evolve into any other configuration states of the mechanism.

Suppose a metamorphic mechanism has a total of $n$ configuration states. The degree of freedom of the mechanism in configuration state $p$ is denoted as $F_{\mathrm{p}}$, and the motion screw system of the mechanism in configuration state $p,\left\{p \$^{m}\right\}$, is denoted as $\left\{p \$^{m} \mid\left(p \$_{1}^{m}, p \$_{2}^{m}, \ldots, p \$_{F_{p}}^{m}\right)\right\}$. Because the reciprocal product of two inverse screws is zero
[12], the constraint screw system of the mechanism in configuration state $p,\left\{p \$^{r}\right\}$, can be denoted as $\left\{p \$^{r} \mid\left(p \$_{1}^{r}, p \$_{2}^{r}, \ldots, p \$_{6-F_{p}}^{r}\right)\right\}$. Therefore, the constraint screw system of branch $L$ of the mechanism, $\left\{p \$_{L}^{r}\right\}$, can be expressed as $\left\{p \$_{L}^{r} \mid\left(p \$_{L, 1}^{r}, p \$_{L, 2}^{r}, \ldots, p \$_{L, i_{p}}^{r}\right)\right\}$.

Here, $i_{p} \leq 6-F_{p}$, where $i_{p}$ is the number of constraint screws of branch $L$ in configuration state $p$ and $\left\{p \$_{L}^{r}\right\}$ should be a subset of $\left\{p \$^{r}\right\}$, that is [12], 


$$
\left\{p_{\$_{L, 1}^{r},}{ }^{p} \oiint_{L, 2}^{r}, \ldots,{ }^{p} \$_{L, i_{p}}^{r}\right\} \subseteq\left\{p_{\$_{1}}^{r}, p_{\$_{2}}^{r}, \ldots,{ }^{p} \$_{6-F_{p}}^{r}\right\}
$$

In configuration state $p$, the constraint screw system of the mechanism should be the union of the constraint screw systems of all branches [12] (suppose the mechanism has a total of $k$ branches), that is,

$$
\bigcup_{L=1}^{k}\left\{p \$_{L, 1}^{r},{ }^{p} \$_{L, 2}^{r}, \ldots,{ }^{p} \$_{L, i_{p}}^{r}\right\}=\left\{p \$_{1}^{r},{ }^{p} \$_{2}^{r}, \ldots,{ }^{p} \$_{6-F_{p}}^{r}\right\}
$$

According to formula (2), the constraint screw system $\left\{p \$_{L}^{r}\right\}$ of branch $L$ in configuration state $p$ can be obtained.

It can be further seen that the union of the motion screw systems of the mechanism in all $n$ configuration states is the motion screw system $\left\{m \$^{s}\right\}$ in full configuration state [15] (herein referred to as the $s$ configuration state), that is,

$$
\bigcup_{p=1}^{n}\left\{p \$^{m}\right\}=\left\{{ }^{s} \$^{m} \mid\left({ }^{s} \$_{1}^{m},{ }^{s} \$_{2}^{m}, \ldots,{ }^{s} \$_{F_{s}}^{m}\right)\right\}
$$

where $F_{s}$ is the degree of freedom of the mechanism in full configuration.

Solving the inverse screw of $\left\{s \$^{m}\right\}$, the constraint screw system $\left\{s \$^{r}\right\}$ of the mechanism in full configuration state can be determined as $\left\{\left\{s \$^{r}\right\} \mid\left({ }^{s} \$_{1}^{r}, s \$_{2}^{r}, \ldots, s \$_{6-F_{s}}^{r}\right)\right\}$. Furthermore, the constraint screw system $\left\{s \$_{L}^{r}\right\}$ of branch $L$ in full configuration state can be determined as $\left\{s \$_{L}^{r} \mid\left({ }^{s} \$_{L, 1}^{r},{ }^{s} \$_{L, 2}^{r}, \ldots,{ }^{s} \$_{L, i_{s}}^{r}\right)\right\}$, where $i_{s} \leq 6-F_{s}$ is the number of constraint screws of branch $L$ in full configuration state and $\left\{s \$_{L}^{r}\right\}$ should be a subset of $\left\{s \$^{r}\right\}$, that is,

$$
\left\{{ }^{s} \$_{L, 1}^{r},{ }^{s} \oiint_{L, 2}^{r}, \ldots,{ }^{s} \$_{L, i_{s}}^{r}\right\} \subseteq\left\{{ }^{s} \$_{1}^{r},{ }^{s} \$_{2}^{r}, \ldots,{ }^{s} \$_{6-F_{s}}^{r}\right\} .
$$

In full configuration state, the constraint screw system $\left\{s \$^{r}\right\}$ of the mechanism should be the union of the constraint screw systems of all branches [12] (suppose the mechanism has a total of $k$ branches), that is,

$$
\bigcup_{L=1}^{k}\left\{{ }^{s} \$_{L, 1}^{r},{ }^{s} \$_{L, 2}^{r}, \ldots,{ }^{s} \$_{L, i_{s}}^{r}\right\}=\left\{{ }^{s} \$_{1}^{r},{ }^{s} \$_{2}^{r}, \ldots,{ }^{s} \$_{6-F_{s}}^{r}\right\}
$$

According to formula (5), the constraint screw system $\left\{s \$_{L}^{r}\right\}$ of branch $L$ in full configuration state can be obtained.

From formula (3), $\left\{p \$^{m}\right\} \subseteq\left\{s \$^{m}\right\}$ is known, and it can be derived according to the dual relationship between constraint and degree of freedom that

$$
\left\{{ }^{s} \$^{r}\right\} \subseteq\left\{p \$^{r}\right\}
$$

It can be derived from formula (6):

$$
\left\{{ }^{s} \$^{r}\right\} \cup\left\{{ }^{p} \$^{r+}\right\}=\left\{{ }^{p} \$^{r}\right\} .
$$

From formula (2), we know that $\cup_{L=1}^{k}\left\{p \$_{L}^{r}\right\}=\left\{p \$^{r}\right\}$; from formula (5), we know that $\cup_{L=1}^{k}\left\{{ }^{s} \$_{L}^{r}\right\}=\left\{{ }^{s} \$^{r}\right\}$; combining with formula (6), we can know

$$
\left\{{ }^{s} \$_{L}^{r}\right\} \subseteq\left\{p \$_{L}^{r}\right\}
$$

Formula (7) shows that, in constraint screw systems $\left\{s \$^{r}\right\}$ of the mechanism in full configuration state (namely, configuration state $s$ ), if the missing constraint screws $\left\{p \oiint^{r+}\right\}$ in full configuration state can be supplemented from those in configuration state $p$, the constraints of the mechanism in full configuration state will increase and the degrees of freedom will reduce. Finally, the mechanism can accurately switch from full configuration state to configuration state $p$ (refer to Section 4 of this article for details).

In summary, full configuration state can be regarded as the initial configuration state of the metamorphic mechanism, which contains all kinds of degrees of freedom in all configuration states. Full configuration can transform into any other configuration states by increasing constraints $\left\{p \$^{r+}\right\}$ (equivalent to reducing degrees of freedom), as shown in formula (7).

The flowchart of configuration synthesis for metamorphic parallel mechanism is proposed based on the principle described above, as shown in Figure 3.

\section{Mechanism Design of Metamorphic Parallel Leg}

4.1. Configuration Synthesis of Branches in Full Configuration State of Metamorphic Mechanism. The metamorphic parallel leg mechanism in this paper has three identical branches. There are two configuration states of the metamorphic parallel leg mechanism, which are $R^{x} R^{y} T^{z}$ (three-degree-offreedom) and $3 R T^{Z}$ (four-degree-of-freedom) configuration states. In the fixed coordinate system of the metamorphic mechanism, ${ }^{1} \$^{m}$ and ${ }^{2} \$^{m}$, the motion screw systems of the mechanism in $R^{x} R^{y} T^{\varepsilon}$ and $3 R T^{z}$ configuration states are, respectively, as follows:

$$
\begin{aligned}
& { }^{1} \oiint^{m}=\left\{\begin{array}{l}
{ }^{1} \$_{1}^{m}=(1,0,0 ; 0,0,0), \\
{ }^{1} \$_{2}^{m}=(0,1,0 ; 0,0,0), \\
{ }^{1} \$_{3}^{m}=(0,0,0 ; 0,0,1),
\end{array}\right. \\
& { }^{2} \oiint^{m}=\left\{\begin{array}{l}
{ }^{2} \$_{1}^{m}=(1,0,0 ; 0,0,0), \\
{ }^{2} \$_{2}^{m}=(0,1,0 ; 0,0,0), \\
{ }^{2} \$_{3}^{m}=(0,0,1 ; 0,0,0), \\
2 \$_{4}^{m}=(0,0,0 ; 0,0,1),
\end{array}\right.
\end{aligned}
$$

The motion screw system ${ }^{s} \$^{m}$ of the metamorphic mechanism in full configuration state can be obtained by substituting formula (9) into formula (3):

$$
{ }^{s} \oiint^{m}=\left\{\begin{array}{c}
s \$_{1}^{m}=(1,0,0 ; 0,0,0), \\
s \$_{2}^{m}=(0,1,0 ; 0,0,0), \\
s \$_{3}^{m}=(0,0,1 ; 0,0,0), \\
s \$_{4}^{m}=(0,0,0 ; 0,0,1) .
\end{array}\right.
$$

It can be seen from formula (10) that the motion screw system in full configuration state (namely, configuration state $s$ ) is exactly the one in $3 R T^{z}$ configuration state. Here, $R^{x} R^{y} T^{z}$ configuration state of the metamorphic mechanism is denoted as configuration state $p$. According to the theory of reciprocal screws [12], it can be deduced as 


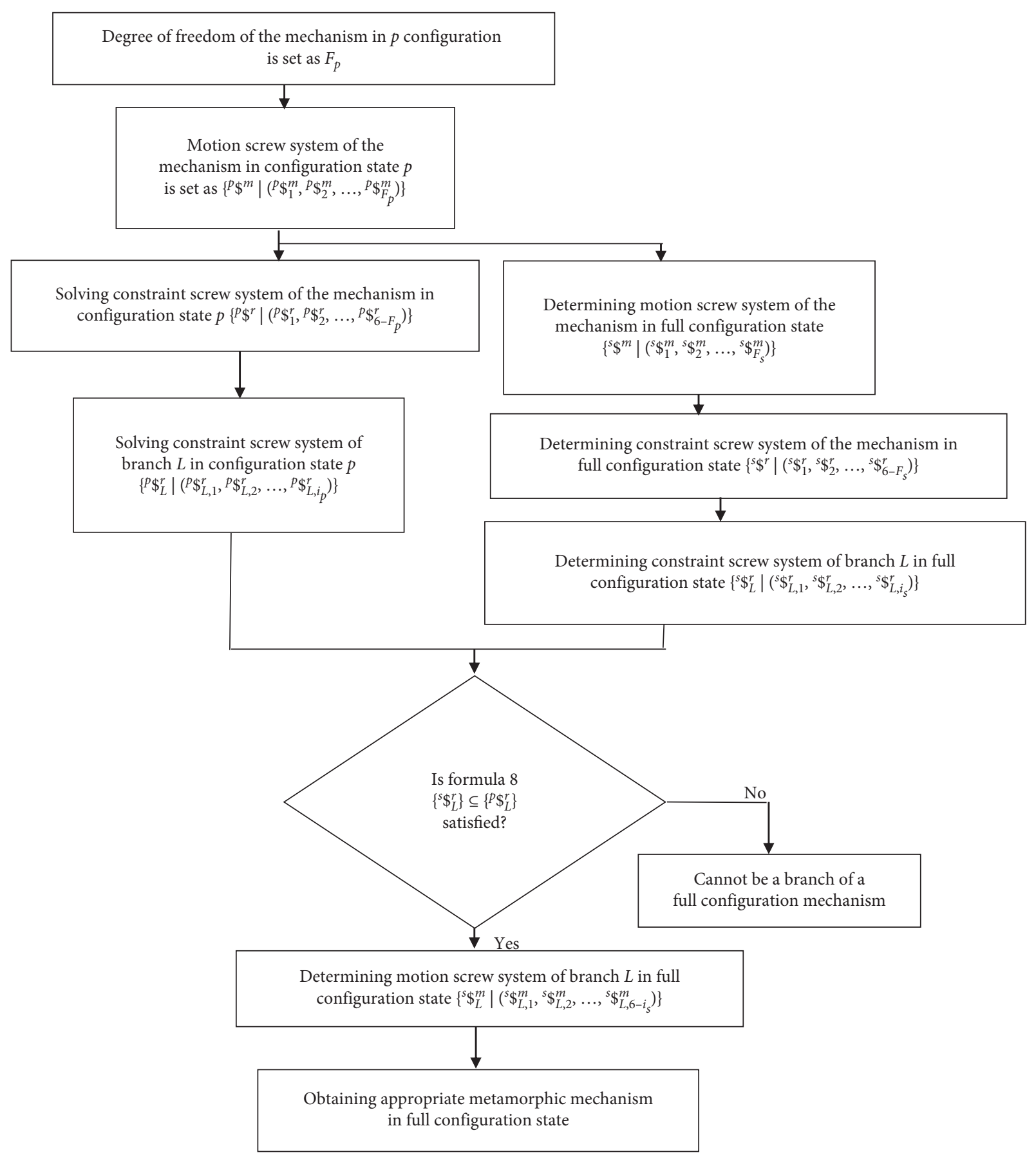

FIGURE 3: Configuration synthesis process of metamorphic parallel mechanism in full configuration state.

$$
\begin{gathered}
p \oiint^{r}=\left\{\begin{array}{l}
p \$_{1}^{r}=(1,0,0 ; 0,0,0), \\
p \$_{2}^{r}=(0,1,0 ; 0,0,0), \\
p \$_{3}^{r}=(0,0,0 ; 0,0,1),
\end{array}\right. \\
{ }^{s} \oiint^{r}=\left\{\begin{array}{l}
s \$_{1}^{r}=(1,0,0 ; 0,0,0), \\
s \oiint_{2}^{r}=(0,1,0 ; 0,0,0),
\end{array}\right.
\end{gathered}
$$

4.1.1. Constraint Screws of Branches in Configuration State $p$ of $R^{x} R^{y} T^{z}$ Metamorphic Mechanism. According to formula
(11), the constraint screws of branches with $R^{x} R^{y} T^{\varepsilon}$ (two rotational and one translational degrees of freedom) contain three cases: the first kind of branch has three degrees of freedom, and it is constrained by two forces and one couple. The second kind of branch has four degrees of freedom and is constrained by one force and one couple. The third kind of branch has five degrees of freedom and is constrained by one force. The specific analyses are as follows.

Two constraint forces and one constraint couple in the constraint screw system of the three-degree-of-freedom branch $L$ here are set as 


$$
\begin{aligned}
& p \$_{L, 1}^{r}=(1,0,0 ; 0,0,0), \\
& p \$_{L, 2}^{r}=(0,1,0 ; 0,0,0), \\
& p \$_{L, 3}^{r}=(0,0,0 ; 0,0,1) .
\end{aligned}
$$

Solving the inverse screws of the constraint screws in formula (13), the motion screw system of branch $L$ can be obtained [12] as

$$
\begin{aligned}
& p \$_{L, 1}^{m}=(1,0,0 ; 0,0,0), \\
& p \$_{L, 2}^{m}=(0,1,0 ; 0,0,0), \\
& p \$_{L, 3}^{m}=(0,0,0 ; 0,0,1) .
\end{aligned}
$$

In this same way, one force and one couple in the constraint screw system of the four-degree-of-freedom branch $L$ can be set as

$$
\begin{aligned}
& p_{\$_{L, 1}^{r}}^{r}=(1,0,0 ; 0,0,0), \\
& p_{\$_{L, 2}^{r}}^{r}=(0,0,0 ; 0,0,1) .
\end{aligned}
$$

Solving the inverse screws of formula (15), the motion screw system of branch $L$ can be obtained as

$$
\begin{gathered}
p_{\$_{L, 1}^{m}}^{m}=(1,0,0 ; 0,0,0), \\
p_{\$_{L, 2}^{m}}=(0,1,0 ; 0,0,0), \\
{ }^{p} \oiint_{L, 3}^{m}=(0,0,0 ; 0,1,0), \\
p_{\$_{L, 3}^{m}}=(0,0,0 ; 0,0,1) .
\end{gathered}
$$

When the degrees of freedom of branch $L$ is three or four, the linear combination of the motion screws $p \$_{L, 1}^{m}$ and $p \$_{L, 2}^{m}$ in formulas (14) or (16) can be expressed as a compound motion screw $\left(l_{L}, m_{L}, 0 ; 0,0,0\right)$, it corresponds to a new rotational pair, and the rotational axis is parallel to the plane of the fixed platform. Obviously, when the moving platform rotates around the $x$ or $y$ axis, the rotational axis of the motion screw $\left(l_{L}, m_{L}, 0 ; 0,0,0\right)$ determined by $p \$_{L, 1}^{m}$ and $p \$_{L, 2}^{m}$ will not always be parallel to the plane of the fixed platform. Therefore, any branches determined by $p \$_{L, 1}^{m}$ and $P \$_{L, 2}^{m}$ can only constitute an instantaneous mechanism, but not a mechanism with stable $R^{x} R^{y} T^{\chi}$ degrees of freedom.

As mentioned above, a five-degree-of freedom branch is constrained by one force, which can be set as

$$
p_{\oiint_{L, 1}^{r}}^{r}=(1,0,0 ; 0,0,0) .
$$

Solving the inverse screws of formula (17), the motion screw system of branch $L$ can be obtained as

$$
\begin{gathered}
p_{\$_{L, 1}^{m}}=(1,0,0 ; 0,0,0), \\
p_{\$_{L, 2}^{m}}=(0,1,0 ; 0,0,0), \\
p_{\$_{L, 3}^{m}}=(0,0,1 ; 0,0,1), \\
p_{\$_{L, 4}^{m}}=(0,0,0 ; 0,1,0), \\
p_{\$_{L, 5}^{m}}^{m}=(0,0,0 ; 0,0,1) .
\end{gathered}
$$

According to formula (18), the branch contains a $2 R$ or $3 R$ spherical subchain, and its center point is set as the center point of the branch in this paper. According to formulas (17) and (18), the constraint force vector of the five-degree-offreedom branch is parallel to $p \$_{L, 1}^{m}$ and passes through the center point of the branch. All the constraint forces $p \$_{L}^{r}$ $(L=1,2,3)$ imposed on branches must be coplanar, but these constraint forces can neither converge at a point (that is, the central points $N_{1}, N_{2}$, and $N_{3}$ of branches do not converge at the same point, as shown in Figure 1(b)) nor be parallel to each other to ensure that the mechanism has $R^{x} R^{y} T^{z}$ degrees of freedom (see Section 5.2. for the specific analysis), so all the center points of branches do not intersect at a point.

4.1.2. Constraint Screws of Branches in Full Configuration State of $3 R T^{\complement}$ Metamorphic Mechanism. According to formula (12), the constraint screws of branches with $3 R T^{Z}$ (three rotational and one translational degrees of freedom) contain two cases: the first kind of branch has four degrees of freedom, and it is constrained by two forces. The second kind of branch has five degrees of freedom and is constrained by one force.

Two constraint forces in the constraint screw system of the four-degree-of-freedom branch $L$ here are set as

$$
\begin{aligned}
& s \oiint_{L, 1}^{r}=(1,0,0 ; 0,0,0), \\
& s \oiint_{L, 2}^{r}=(0,1,0 ; 0,0,0) .
\end{aligned}
$$

Solving the inverse screws of the constraint screws in formula (19), the motion screw system of branch $L$ can be obtained [12] as

$$
\begin{aligned}
& s \oiint_{L, 1}^{m}=(1,0,0 ; 0,0,0), \\
& s \oiint_{L, 2}^{m}=(0,1,0 ; 0,0,0), \\
& s \oiint_{L, 3}^{m}=(0,0,1 ; 0,0,0), \\
& s \oiint_{L, 4}^{m}=(0,0,0 ; 0,0,1) .
\end{aligned}
$$

One force in the constraint screw system of the fivedegree-of-freedom branch $L$ here is set as

$$
s \$_{L, 1}^{r}=(1,0,0 ; 0,0,0) .
$$

Solving the inverse screws of formula (21), the motion screw system of branch $L$ can be obtained as

$$
\begin{gathered}
{ }^{s} \$_{L, 1}^{m}=(1,0,0 ; 0,0,0), \\
{ }^{s} \$_{L, 2}^{m}=(0,1,0 ; 0,0,0), \\
{ }^{s} \oiint_{L, 3}^{m}=(0,0,1 ; 0,0,1), \\
{ }^{s} \oiint_{L, 4}^{m}=(0,0,0 ; 0,1,0), \\
{ }^{s} \oiint_{L, 5}^{m}=(0,0,0 ; 0,0,1) .
\end{gathered}
$$

According to formulas (20) and (22), the branch contains a $2 R$ or $3 R$ spherical subchain, and its center point is set as the center point of the branch in this paper. According to formulas (19) and (21), the constraint force vectors of each branch are parallel to the plane of the fixed platform and passes through the center point of the branch. All the constraint forces $s \$_{L}^{r}(L=1,2,3)$ imposed on branches must be coplanar and converge at the same point $N$ (as shown in Figure 2(b)) to ensure that the mechanism has $3 R T^{z}$ degrees of freedom (see Section 5.1 for the specific analysis). In order 
to ensure that the center points of all branches always converge at the same point during mechanism motion, one of the revolute pairs in $2 R$ or $3 R$ spherical subchain of the branch should be connected with the moving platform.

4.1.3. Configuration Synthesis of Branches in Full Configuration State of Metamorphic Mechanism. As known in Section 4.1.1, the constraint screw of branches in configuration state $p$ of the mechanism with $R^{x} R^{y} T^{z}$ degrees of freedom is

$$
p_{\$_{L, 1}^{r}}^{r}=(1,0,0 ; 0,0,0)
$$

As known in Section 4.1.2, the constraint screws of branches in full configuration state of the mechanism with $3 R T^{\complement}$ degrees of freedom are

$$
\begin{array}{r}
{ }^{s} \$_{L, 1}^{r}=(1,0,0 ; 0,0,0) \\
\left\{\begin{array}{c}
{ }^{s} \$_{L, 1}^{r}=(1,0,0 ; 0,0,0), \\
{ }^{s} \oiint_{L, 2}^{r}=(0,1,0 ; 0,0,0) .
\end{array}\right.
\end{array}
$$

According to the constraint screw principle [12] and switching condition in formula (7) from $s$ configuration state to $p$ configuration state, the results of configuration synthesis of branches in full configuration state of the metamorphic mechanism are shown in Table 1.

In Table 1, $v$ indicates that the axis of the kinematic pair is parallel to the plane of the fixed platform, $u$ indicates that the axis of the kinematic pair intersects but is not perpendicular to the plane of the fixed platform, and $\left({ }^{i} R^{j} R^{k} R\right)$ indicates that axes $i, \mathrm{j}$, and $k$ intersect at a point. In this paper, the full-configuration branch ${ }^{v} R^{u} P^{v} R\left({ }^{j} R^{k} R\right)$ is selected to construct the metamorphic mechanism.

\subsection{Design of Metamorphic Mechanism with Reconfigurable} Moving Platform. According to the metamorphic principle [15], the linear independence of the constraint screws can be obtained by properly arranging the spatial geometric relations among them, which can change the degree of freedom and transform configuration states of metamorphic mechanism.

Analysis of Table 1 shows that, in $s$ configuration state of the mechanism with $3 R T^{\mathcal{L}}$ degrees of freedom, the center points of branches converge at a point and the constraint screws of branches are linear dependent, and that, in $p$ configuration state of $R^{x} R^{y} T^{z}$ mechanism, the center points of branches do not converge at a point and the constraint screws of branches are linear independent. The relative position of the center points of branches can be changed by transforming the axes direction of kinematic pairs in branches, and thus, switching between configuration states of the mechanism is realized. In this paper, a triple symmetric Bricard mechanism as the configurable moving platform is used to realize the switching between configuration states of the metamorphism parallel mechanism. Symmetric Bricard Mechanism. The triple symmetric
Bricard mechanism in Figure 4 is a single-degree-of-freedom spatial closed-loop mechanism. The mechanism has three symmetry planes, which are determined, respectively, by the axes of revolute pairs $R_{1}$ and $R_{4}, R_{2}$ and $R_{5}$, and $R_{3}$, and $R_{6}$. When the mechanism moves, the axes of revolute pairs are always located in their respective symmetric planes [16]. According to the triple symmetry property of Bricard mechanism, the corresponding geometric parameters should meet the following conditions during mechanism movement:

$$
\begin{aligned}
\theta_{1} & =\theta_{3}=\theta_{5}=\theta, \\
\theta_{2} & =\theta_{4}=\theta_{6}=\varphi, \\
a_{12} & =a_{23}=a_{34}=a_{45}=a_{56}=a_{61}=a, \\
\alpha_{12} & =\alpha_{34}=\alpha_{56}=\alpha, \alpha_{23}=\alpha_{45}=\alpha_{61}=2 \pi-\alpha .
\end{aligned}
$$

In formula (25), $\theta_{i}(i=1,2, \ldots, 6)$ is the rotation angle of revolute pair $i$. $a_{12}, a_{23}, a_{34}, a_{45}, a_{56}$, and $a_{61}$ is, respectively, the link length of the triple symmetric Bricard mechanism, and $\alpha_{12}, \alpha_{34}, \alpha_{56}, \alpha_{23}, \alpha_{45}$, and $\alpha_{61}$ is, respectively, angle between the axes of two revolute pairs at both ends of a link. The usual rigid moving platform is replaced by the triple symmetrical Bricard mechanism in this paper, and three nonadjacent revolute pairs $\left(R_{1}, R_{3}\right.$, and $R_{5}$ or $R_{2}, R_{4}$, and $\left.R_{6}\right)$ of the Bricard mechanism are used to connect with three branches of the metamorphic parallel leg mechanism.

4.2.2. Design of the Metamorphic Parallel Mechanism with a Reconfigurable Moving Platform. In this paper, revolute pairs $R_{2}, R_{4}$, and $R_{6}$ of the Bricard mechanism in Figure 4 are selected to connect with the three branches of the metamorphic parallel mechanism; that is, the revolute pairs $R_{2}$, $R_{4}$, and $R_{6}$ are the ${ }^{k} R$ revolute pairs of ${ }^{v} R^{v} R^{u} P\left({ }^{j} R^{k} R\right)$ branches in full configuration state of the metamorphic parallel leg mechanism.

When the robot climbs stairs, the parallel leg mechanism has $3 R T^{\complement}$ degrees of freedom (the mechanism is in the $s$ configuration state), and in the corresponding Bricard mechanism, the axes of $R_{1}, R_{3}$, and $R_{5}$ are parallel to each other, and the axes of $R_{2}, R_{4}$, and $R_{6}$ converge at a point, as shown in Figure 5(a). At the time, the center points of three branches of the parallel mechanism converge at the point $\mathrm{N}$, as shown in Figure 2(b).

When the robot walks on flat ground, the parallel leg mechanism has $R^{x} R^{y} T^{z}$ degrees of freedom (the mechanism is in configuration state $p$ ), and in the corresponding Bricard mechanism, the axes of $R_{2}, R_{4}$, and $R_{6}$ are parallel to each other, and the axes of $R_{1}, R_{3}$, and $R_{5}$ converge at a point, as shown in Figure 5(b). At the time, the center points of three branches of the parallel mechanism do not converge at a point, as shown in Figure 1(b).

The Bricard mechanism, which is actuated by its driving pair $R_{1}$ in this paper, is exactly the reconfigurable moving platform of the metamorphic parallel mechanism. The relative motions between links in the Bricard mechanism change the geometric relationships between axes of the kinematic pairs in each branch of the metamorphic parallel mechanism and change the position of the center point of 
TABle 1: Configuration synthesis results of branches in full configuration state.

\begin{tabular}{|c|c|c|c|c|c|}
\hline $\begin{array}{l}\text { Constraint screws of } \\
\text { branches in } p \\
\text { configuration state of } \\
R^{x} R^{y} T^{z} \text { mechanism }\end{array}$ & $\begin{array}{l}\text { Constraint screws of } \\
\text { branches in } s \text { configuration } \\
\text { state of } 3 R T^{\varepsilon} \text { mechanism }\end{array}$ & $\begin{array}{c}\text { Is } \\
\left\{s \$_{L}^{r}\right\} \subseteq\left\{p \$_{L}^{r}\right\} \\
\text { satisfied? }\end{array}$ & $\begin{array}{c}\text { Branch } \\
\text { configurations in } \\
\text { full configuration } \\
\text { state }\end{array}$ & $\begin{array}{l}\text { Branch assembly } \\
\text { conditions in } p \\
\text { configuration state } \\
\text { of } R^{x} R^{y} T^{\varepsilon} \\
\text { mechanism }\end{array}$ & $\begin{array}{l}\text { Branch assembly } \\
\text { conditions in } s \\
\text { configuration state of } \\
3 R T^{\varepsilon} \text { mechanism }\end{array}$ \\
\hline$p \$_{L, 1}^{r}=(1,0,0 ; 0,0,0)$ & $\left\{\begin{array}{l}{ }^{s} \$_{L, 1}^{r}=(1,0,0 ; 0,0,0) \\
s s \$_{L, 2}^{r}=(0,1,0 ; 0,0,0)\end{array}\right.$ & Yes & $\begin{array}{l}{ }^{v} R^{u} P\left({ }^{i} R^{j} R^{k} R\right) \\
{ }^{u} P^{v} R\left({ }^{i} R^{j} R^{k} R\right) \\
{ }^{v} R^{v} R\left({ }^{i} R^{j} R^{k} R\right) \\
{ }^{v} R^{v} R^{v} R\left({ }^{j} R^{k} R\right) \\
{ }^{u} P^{v} R^{v} R\left({ }^{j} R^{k} R\right) \\
{ }^{v} R^{v} R^{u} P\left({ }^{j} R^{k} R\right) \\
{ }^{v} R^{u} P^{v} R\left({ }^{j} R^{k} R\right)\end{array}$ & $\begin{array}{l}\text { (1) Center points of } \\
\text { branches does not } \\
\text { converge at a point } \\
\text { (2) Rotational axes } \\
\text { corresponding to } \\
p \$_{L, 1}^{m} \text { of each branch } \\
\text { are not parallel to } \\
\text { each other }\end{array}$ & $\begin{array}{c}\text { (1) Center points of } \\
\text { branches converge at } \\
\text { a point } \\
\text { (2) One of revolute } \\
\text { pairs in } 2 R \text { or } 3 R \\
\text { spherical subchain of } \\
\text { the branch connects } \\
\text { with the moving } \\
\text { platform }\end{array}$ \\
\hline
\end{tabular}

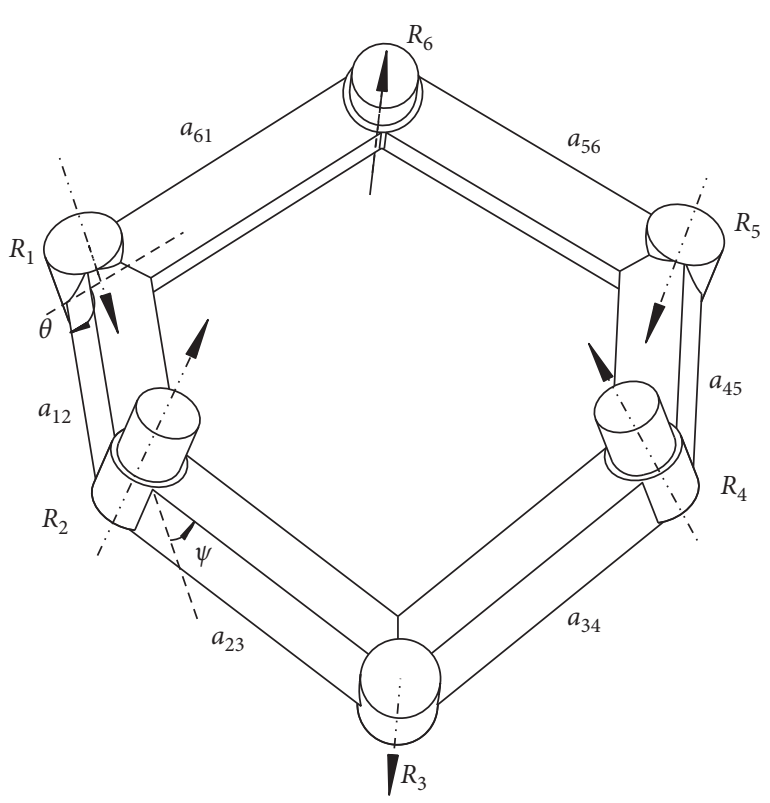

FIgURE 4: Triple symmetrical Bricard mechanism.

each branch. As a result, the configuration state of the metamorphic parallel leg mechanism is switched from $3 R T^{\varepsilon}$ in Figure 6 to $R^{x} R^{y} T^{z}$ in Figure 7 .

As mentioned above, revolute pairs $R_{2}, R_{4}$, and $R_{6}$ of the Bricard mechanism is, respectively, connected with each branch of the metamorphic parallel mechanism. Therefore, the motion of the Bricard mechanism can change the relative position of the center points of branches of the metamorphic parallel mechanism. After the geometric relations between axes of the revolute pairs in the Bricard mechanism and the geometric relations between center points of the branches of the metamorphic parallel mechanism with desired degree of freedom are satisfied (as shown in Figures 5-7), rigidizing the driving pair of Bricard mechanism (i.e., make Bricard mechanism a rigid moving platform of parallel leg mechanism), the configuration state transformation of the metamorphic parallel leg mechanism can be completed.

The branch ${ }^{v} R^{u} P^{v} R\left({ }^{j} R^{k} R\right)$ in Table 1 was selected as the metamorphic branch in full configuration state. According to the assembly requirements of mechanism branches in two configuration states in Table 1, by combining branches with Bricard mechanism (the reconfigurable moving platform), a metamorphic parallel leg mechanism satisfying the requirements can be constructed, as shown in Figures 6 and 7.

In Figure 6, the metamorphic parallel leg mechanism 3${ }^{v} R^{u} P^{v} R\left({ }^{j} R^{k} R\right)_{N}$ is in full configuration state. At this time, the axes of revolute pairs $R_{13}, R_{14}, R_{23}, R_{24}, R_{33}$, and $R_{34}$ of spherical subchains $\left({ }^{j} R^{k} R\right)$ in three branches of the metamorphic mechanism converge at a point $N$. Furthermore, the six axes always converge at a point during movement of the mechanism. In Figure 7, the metamorphic parallel mechanism ${ }^{3-}{ }^{v} R^{u} P^{v} R\left({ }^{j} R^{k} R\right)_{N^{3}}$ is in the $p$ configuration state. The center points $N_{1}, N_{2}$, and $N_{3}$ of the spherical subchains $\left({ }^{j} R^{k} R\right)$ in three branches of this mechanism do not converge at a point.

\section{Verification of Degrees of Freedom of the Metamorphic Parallel Mechanism}

5.1. Verification of Degrees of Freedom of $3-^{v} R^{u} P^{v} R\left({ }^{j} R^{k} R\right)_{N}$ Metamorphic Parallel Mechanism. Motion screws of a branch of $3-^{v} R^{u} P^{v} R\left({ }^{j} R^{k} R\right)_{N}$ parallel mechanism with $3 R T^{Z}$ degrees of freedom are shown in Figure 8 . The center point $O_{L}$ of branches (that is, the center of the spherical subchains) is selected as the origin of the branch coordinate system, the positive direction of the $x_{L}$ axis of the branch coordinate system is parallel to the axis of the revolute pair corresponding to motion screw $\$_{L, 1}$, and the positive direction of the $z_{L}$ axis is perpendicular downwards to the fixed platform. When the moving platform is parallel to the fixed platform, the motion screws of each branch of the mechanism are shown as follows:

$$
\begin{aligned}
& { }^{s} \oiint_{L, 1}^{m}=\left(\begin{array}{lllll}
1 & 0 & 0 & 0 & a_{L 1} \\
b_{L 1}
\end{array}\right),
\end{aligned}
$$

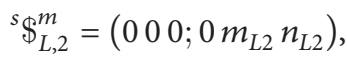

$$
\begin{aligned}
& { }^{s} \$_{L, 3}^{m}=\left(\begin{array}{llll}
1 & 0 & 0 ; 0 & a_{L 3} b_{L 3}
\end{array}\right) \text {, } \\
& { }^{s} \oiint_{L, 4}^{m}=\left(l_{L 4} m_{L 4} n_{L 4} ; 0000\right) \text {, } \\
& s^{s} \$_{L, 5}^{m}=\left(l_{L 5} m_{L 5} n_{L 5} ; 000\right) \text {, }
\end{aligned}
$$

where $a_{L, j}, b_{L, j}, l_{L, j}, m_{L, j}$, and $n_{L, j}$ are all constant. By solving the inverse screws of the motion screws in formula (27), the constraint screw of a branch can be obtained as follows: 


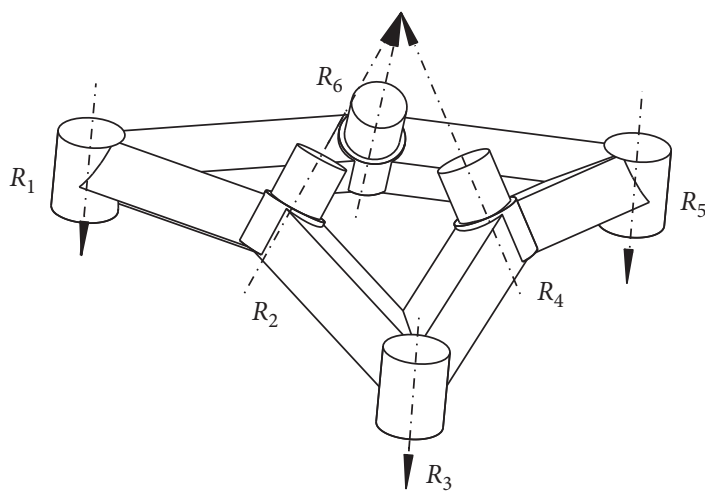

(a)

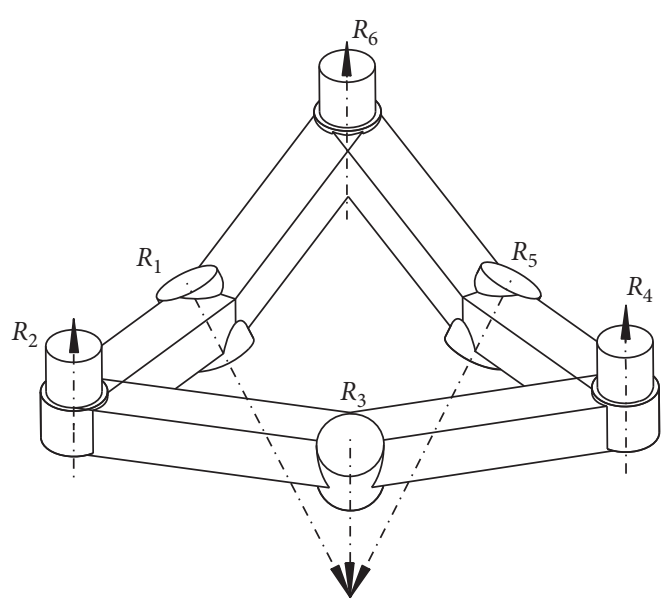

(b)

Figure 5: Bricard mechanisms in different configuration states of the metamorphic parallel mechanism: (a) Bricard mechanism in full configuration state; (b) Bricard mechanism in configuration state $p$.
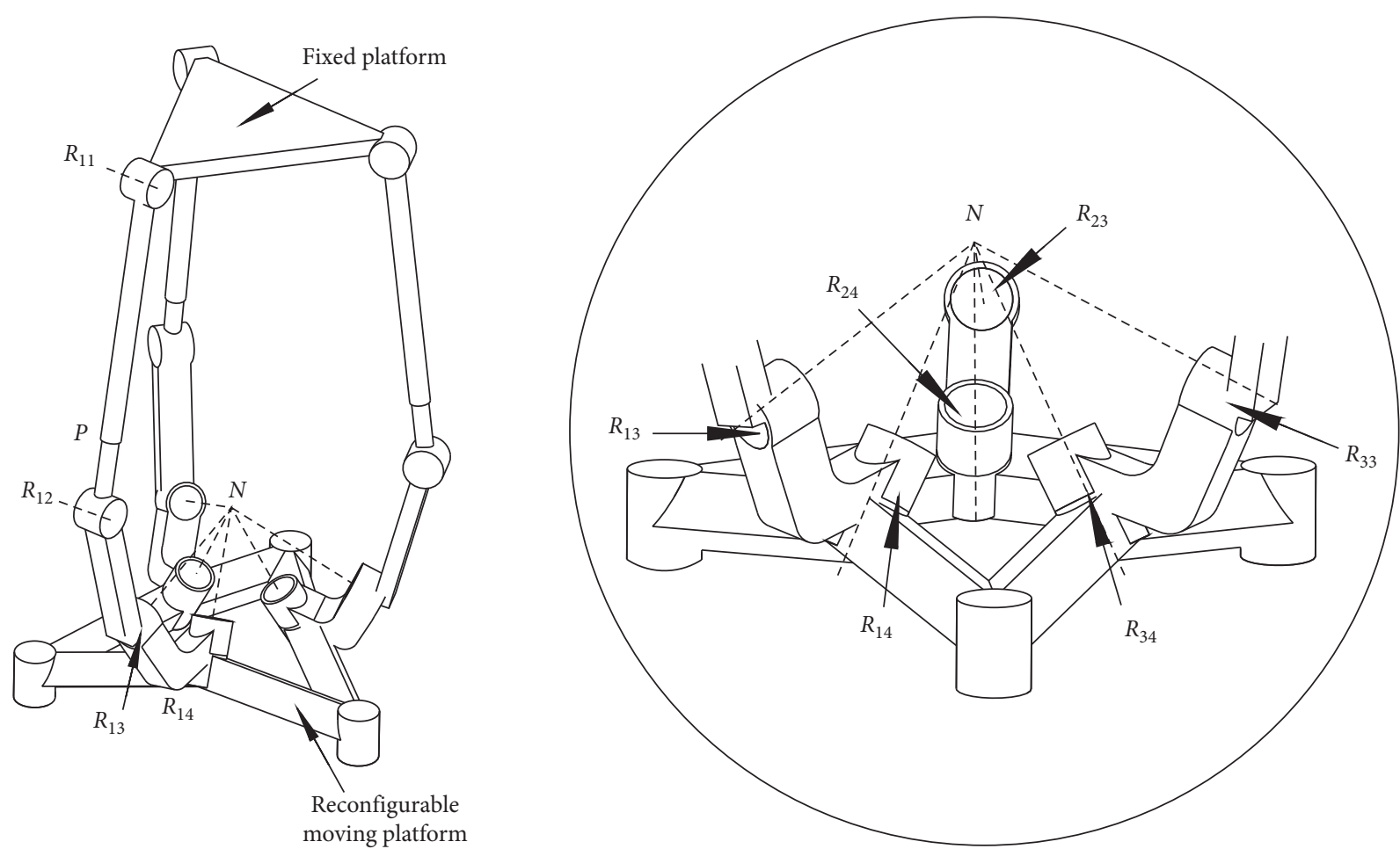

FIgure 6: Metamorphic parallel leg mechanism with $3 R T^{\complement}$ degrees of freedom.

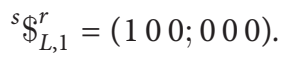

According to formula (27), it can be known that the constraint imposed by a branch on the moving platform is a constraint force vector along the $x_{L}$ axis and through coordinate origin $O_{L}$. The constraint forces of three branches are parallel to the plane of the fixed platform and intersect at one point. So, they are linearly dependent of each other, and their maximum linearly independent number is two; thus, there is one redundant constraint. At this point, the degree of freedom of the mechanism can be calculated according to the modified G-K formula $[17,18]$ :

$$
M=d(n-g-1)+\sum_{1}^{g} f_{i}+v=6(14-15-1)+15+1=4 .
$$

Formulas (27) and (28) verify that the mechanism can meet the requirements of $3 R T_{z}$ degrees of freedom.

5.2. Verification of Degrees of Freedom of $3{ }^{v} R^{u} P^{v} R\left({ }^{j} R^{k} R\right)_{N^{3}}$ Metamorphic Parallel Mechanism. Motion screws of a branch of $3-^{v} R^{u} P^{v} R\left({ }^{j} R^{k} R\right)_{N^{3}}$ parallel mechanism with $R^{x} R^{y} T^{z}$ degrees of freedom are shown in Figure 9. 


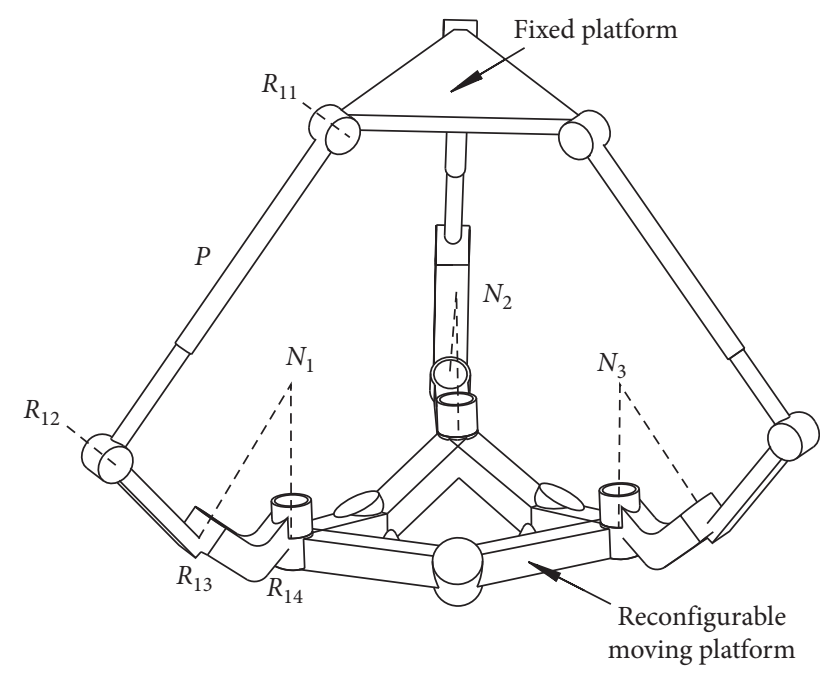

Figure 7: Metamorphic parallel leg mechanism with $R^{x} R^{y} T^{\chi}$ degrees of freedom.

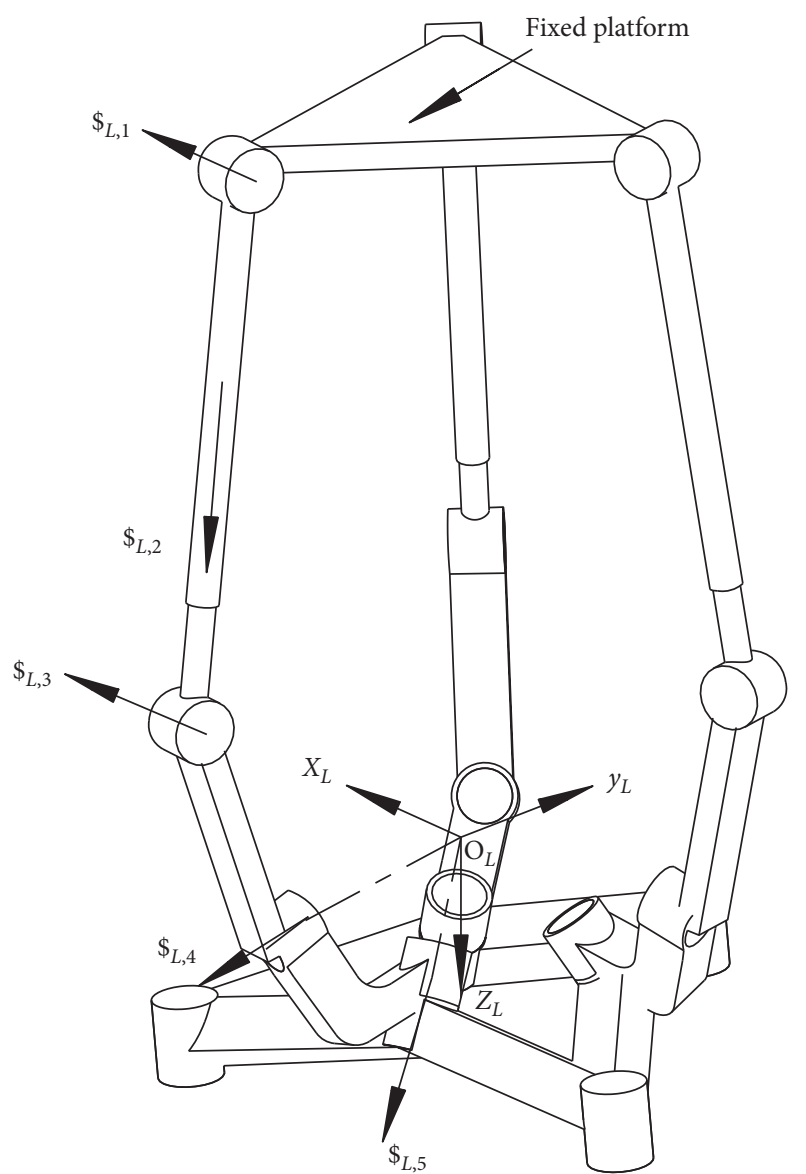

FiguRe 8: Motion screws of a branch of $3-^{v} R^{u} P^{v} R\left({ }^{j} R^{k} R\right)_{N}$ parallel mechanism.

The center point $O_{L}$ of the branch (that is, the center of the spherical subchain of the branch) is selected as the origin of the branch coordinate system, the positive direction of the $x_{L}$ axis of the branch coordinate system is parallel to the axis of the revolute pair corresponding to motion screw $\$_{L, 1}$, and

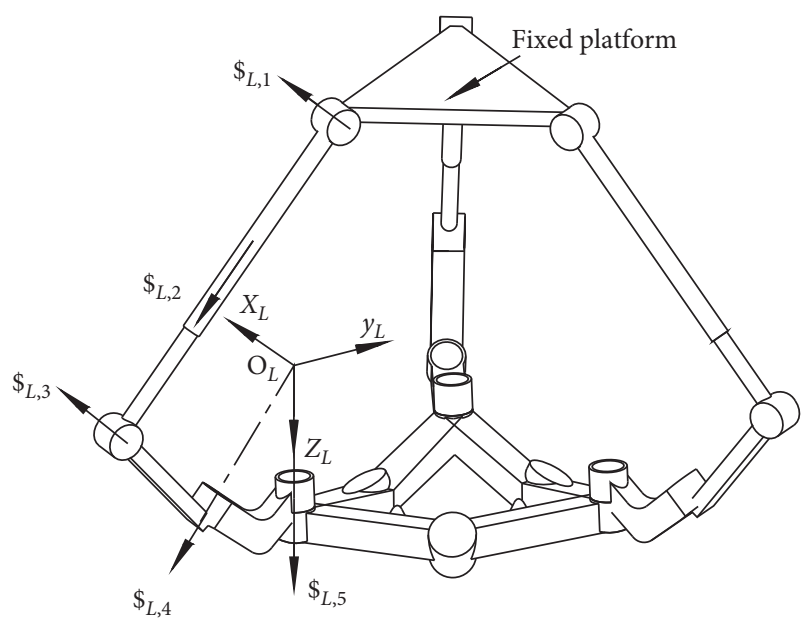

FIgURE 9: Motion screws of a branch of $3{ }^{v} R^{u} P^{v} R\left({ }^{j} R^{k} R\right)_{N^{3}}$ parallel mechanism.

the positive direction of the $z_{L}$ axis is perpendicular downwards to the fixed platform. The motion screws of each branch of the mechanism are shown as follows:

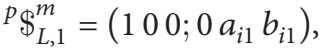

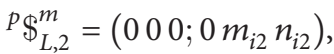

$$
\begin{aligned}
& { }^{p} \$_{L, 3}^{m}=\left(\begin{array}{llllll}
1 & 0 & 0 & 0 & a_{i 3} & b_{i 3}
\end{array}\right), \\
& { }^{p} \$_{L, 4}^{m}=\left(l_{i 4} m_{i 4} n_{i 4} ; 0000\right) \text {, }
\end{aligned}
$$

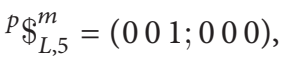

where $a_{L, j}, b_{L, j}, l_{L, j}, m_{L, j}$, and $n_{L, j}$ are all constant. By solving the inverse screws of the motion screws in formula (29), the constraint screw of a branch can be obtained as follows:

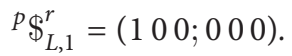

According to formula (30), it can be known that the constraint imposed by a branch on the moving platform is a constraint force line vector along the $x_{L}$ axis and through coordinate origin $O_{L}$. The constraint forces of three branches are parallel to the plane of the fixed platform but do not intersect at one point. So they are linearly independent of each other, and there is no redundant constraint. At this point, the degree of freedom of the mechanism can be calculated according to the modified $\mathrm{G}-\mathrm{K}$ formula:

$$
M=d(n-g-1)+\sum_{1}^{g} f_{i}+v=6 \times(14-15-1)+15=3 .
$$

Formulas (30) and (31) verify that the mechanism can meet the requirements of $R^{x} R^{y} T^{z}$ degrees of freedom.

\section{Conclusions}

This paper puts forward the configuration synthesis principle of metamorphic parallel mechanism based on the concept of full configuration state. The most key issue in the configuration synthesis of metamorphic parallel mechanism 
is that of the mechanism in full configuration state, and then full configuration state evolves into a specific configuration state by increasing constraint or decreasing degrees of freedom. The main contributions in the paper are as follows:

(1) Based on the constraint screw theory, the logical relationships between the constraint screws of the metamorphic parallel mechanism in different configuration states are proposed, and thus, the configuration synthesis principle of metamorphic parallel mechanism is put forward.

(2) A reconfigurable moving platform based on the triple symmetric Bricard spatial closed-loop mechanism with a single degree of freedom is proposed. Based on this, a new method for switching configuration states of the metamorphic parallel mechanism is constructed.

(3) According to the configuration synthesis principle presented in the paper, a novel metamorphic parallel mechanism that can switch between three- and fourdegree-of-freedom configuration states is synthesized, and then the triple-symmetric Bricard spatial closed-chain mechanism is used as the reconfigurable moving platform of the metamorphic mechanism; thus, a novel metamorphic parallel leg mechanism is created.

The above results prove rationality of the configuration design methodology of metamorphic parallel leg mechanism proposed in this paper. The related results lay the necessary theoretical foundation for the further improvement of flexibility and adaptability of walking robots in unstructured environment.

\section{Data Availability}

The research data in the paper are all original data including qualitative and quantitative data. The research data used to support the findings of this study are included within the paper. There is no restriction on data access.

\section{Conflicts of Interest}

The authors declare that they have no conflicts of interest.

\section{Acknowledgments}

This work has been supported by Overseas Visiting Study Program for Young and Middle-Aged Teachers in Universities in Shanghai (2018).

\section{References}

[1] K. T. Zhang, Mechanism Design Method and Performance Study of Metamorphic Parallel Mechanism, Beijing Jiaotong University, Beijing, China, 2010.

[2] D. Li, Z. H. Zhang, J. S. Dai, and K. T. Zhang, "Overview and prospects of metamorphic mechanism," Journal of Mechanical Engineering, vol. 46, no. 13, pp. 14-21, 2010.

[3] Y. G. Wang, J. X. Sun, and J. S. Dai, "Research on metamorphic formula of metamorphic mechanism based on genetic gene theory," Machine Design \& Research, vol. 35, no. 1, pp. 75-80, 2019.

[4] D. Li, J. S. Dai, Q. X. Zhang, and G. G. Jin, "Mobility of a kind of metamorphic mechanism-magic ball," Chinese Journal of Mechanical Engineering, vol. 38, no. 9, pp. 12-16, 2002.

[5] D. Wang and J. S. Dai, "Theoretical foundation of metamorphic mechanism and its synthesis," Chinese Journal of Mechanical Engineering, vol. 43, no. 8, pp. 32-42, 2007.

[6] F. Xi, Y. Xu, and G. Xiong, "Design and analysis of a reconfigurable parallel robot," Mechanism and Machine Theory, vol. 41, no. 2, pp. 191-211, 2006.

[7] W. Ye, Y. Fang, and S. Guo, "Design and analysis of a reconfigurable parallel mechanism for multidirectional additive manufacturing," Mechanism and Machine Theory, vol. 112, pp. 307-326, 2017.

[8] D. Gan, J. Dias, and L. Seneviratne, "Unified kinematics and optimal design of a 3rRPS metamorphic parallel mechanism with a reconfigurable revolute joint," Mechanism and Machine Theory, vol. 96, pp. 239-254, 2016.

[9] D. M. Gan, J. S. Dai, J. Dias et al., "Variable motion/force transmissibility of a metamorphic parallel mechanism with reconfigurable 3T and 3R motion," ASME Journal of Mechanisms and Robotics, vol. 8, Article ID 051001, 2016.

[10] X. Kong and Y. Jin, "Type synthesis of 3-DOF multi-mode translational/spherical parallel mechanisms with lockable joints," Mechanism and Machine Theory, vol. 96, pp. 323-333, 2016.

[11] J. He and F. Gao, "Type synthesis for bionic quadruped walking robots," Journal of Bionic Engineering, vol. 12, no. 4, pp. 527-538, 2015.

[12] Z. Huang, Y. S. Zhao, and T. S. Zhao, Advanced Spatial Mechanism, Higher Education Press, Beijing, China, 2006.

[13] Da Xi and F. Gao, "Type synthesis of walking robot legs," Chinese Journal of Mechanical Engineering, vol. 31, no. 15, pp. 1-13, 2018.

[14] B. W. Gao, J. P. Shao, and G. T. Sun, "Structural design and gait planning of hydraulically actuated quadruped bionic robot," Journal of the Chinese Society of Mechanical Engineers, vol. 36, no. 5, pp. 421-430, 2015.

[15] L. P. Zhang and J. S. Dai, "Metamorphic techniques and geometric reconfiguration principles," in Proceedings of the International Conference on Reconfigurable Mechanisms and Robots, pp. 32-40, London, United Kingdom, June 2009.

[16] Y. Chen, Z. You, and T. Tarnai, "Threefold-symmetric Bricard linkages for deployable structures," International Journal of Solids and Structures, vol. 42, no. 8, pp. 2287-2301, 2004.

[17] Y. P. Lv, Y. Xu, and J. L. Chen, "Optimal design of kinematic performance for a novel 2R1T parallel mechanism with pantograph units," Journal of the Brazilian Society of Mechanical Sciences and Engineering, vol. 41, Article ID 051001, 2019.

[18] Y. Liu, Y. Xu, W. Song, and Y. P. Lv, "Configuration design and position analysis of symmetrical 3T1R parallel mechanisms," Machine Design \& Research, vol. 35, no. 2, pp. 55-59, 2019. 